\title{
Micro-CT Imaging of Tumor Angiogenesis
}

\section{Quantitative Measures Describing Micromorphology and Vascularization}

\author{
Josef Ehling, ${ }^{* \dagger}$ Benjamin Theek, ${ }^{*}$ Felix Gremse, ${ }^{*}$ Sarah Baetke, ${ }^{*}$ Diana Möckel, ${ }^{*}$ Juliana Maynard, ${ }^{\ddagger}$ Sally-Ann Ricketts, \\ Holger Grüll, ${ }^{\S}$ Michal Neeman, ${ }^{\lceil}$Ruth Knuechel, ${ }^{\dagger}$ Wiltrud Lederle, ${ }^{*}$ Fabian Kiessling, ${ }^{*}$ and Twan Lammers ${ }^{*} \|_{* *}$
}

\begin{abstract}
From the Department of Experimental Molecular Imaging, * University Clinic and Helmholtz Institute for Biomedical Engineering, and the Institute of Pathology, ${ }^{\dagger}$ Medical Faculty, RWTH-Aachen University, Aachen, Germany; Personalized Healthcare \& Biomarkers, ${ }^{\ddagger}$ AstraZeneca, Alderley Park, Macclesfield, United Kingdom; the Department of Biomedical Engineering, ${ }^{\S}$ Eindhoven University of Technology, Eindhoven, the Netherlands; the Department of Biological Regulation, "Weizmann Institute of Science, Rehovot, Israel; the Department of Targeted Therapeutics, "MIRA Institute for Biomedical Technology and Technical Medicine, University of Twente, Enschede, the Netherlands; and the Department of Pharmaceutics, ** Utrecht Institute for Pharmaceutical Sciences, Utrecht University, Utrecht, the Netherlands
\end{abstract}

Accepted for publication

October 28, 2013.

Address correspondence to Twan Lammers, Ph.D., D.Sc., or Fabian Kiessling, M.D., Department of Experimental Molecular Imaging, Medical Faculty of the RWTH Aachen, Pauwelsstr. 30, 52074 Aachen, Germany. E-mail: tlammers@ ukaachen.de or fkiessling@ ukaachen.de.

\begin{abstract}
Angiogenesis is a hallmark of cancer, and its noninvasive visualization and quantification are key factors for facilitating translational anticancer research. Using four tumor models characterized by different degrees of aggressiveness and angiogenesis, we show that the combination of functional in vivo and anatomical ex vivo X-ray micro-computed tomography $(\mu \mathrm{CT})$ allows highly accurate quantification of relative blood volume (rBV) and highly detailed three-dimensional analysis of the vascular network in tumors. Depending on the tumor model, $r B V$ values determined using in vivo $\mu \mathrm{CT}$ ranged from $2.6 \%$ to $6.0 \%$, and corresponds well with the values assessed using IHC. Using ultra-high-resolution ex vivo $\mu \mathrm{CT}$, blood vessels as small as $3.4 \mu \mathrm{m}$ and vessel branches up to the seventh order could be visualized, enabling a highly detailed and quantitative analysis of the three-dimensional micromorphology of tumor vessels. Microvascular parameters such as vessel size and vessel branching correlated very well with tumor aggressiveness and angiogenesis. In rapidly growing and highly angiogenic A431 tumors, the majority of vessels were small and branched only once or twice, whereas in slowly growing A549 tumors, the vessels were much larger and branched four to seven times. Thus, we consider that combining highly accurate functional with highly detailed anatomical $\mu \mathrm{CT}$ is a useful tool for facilitating high-throughput, quantitative, and translational (anti-) angiogenesis and antiangiogenesis research. (Am J Pathol 2014, 184: 431-441; http://dx.doi.org/10.1016/j.ajpath.2013.10.014)
\end{abstract}

Angiogenesis, the physiological or pathophysiological process of blood vessel formation and growth, plays an important role in both health and disease. ${ }^{1-3}$ As a classical hallmark of cancer, angiogenesis is essential for enabling tumors to grow beyond a size of 1 to $2 \mathrm{~mm}^{3}$. ${ }^{4,5}$ Consequently, inhibiting angiogenesis has been extensively used as a broadly applicable means for attenuating tumor growth. ${ }^{6}$ Numerous antiangiogenic agents have been evaluated over the years, both in animal models and in patients, and six of these formulations are currently approved for clinical use. , $^{3,7}$

To assess the efficacy of antiangiogenic treatments, functional blood vessels in tumors need to be visualized and quantitatively characterized. At the preclinical level, this is generally done using IHC methods, such as determining the microvessel density in tumors ${ }^{8-10}$ or quantifying the area

\footnotetext{
Supported in part by AstraZeneca UK, German Federal State of North Rhine Westphalia [NRW; HighTech.NRW/EU-Ziel 2-Programm (EFRE); ForSaTum], European Union (European Regional Development Fund-Investing In Your Future; and COST-Action TD1004), European Research Council (ERC Starting Grant 309495-NeoNaNo), German Research Foundation (DFG; EH 412/1-1 and LA 2937/1-2), and RWTH Aachen University (START-152/12).

Disclosures: J.M. and S.-A.R. are employees of AstraZeneca, which provided partial funding for the study.
} 
fraction of $\mathrm{CD} 1^{+}$vessel structures. ${ }^{11,12}$ Although IHC seems to be the most accurate method for assessing tumor angiogenesis, it has several important limitations, in particular its invasive nature and the inability to visualize threedimensional structures.

To overcome these shortcomings, IHC is often supplemented by noninvasive imaging techniques, to provide longitudinal in vivo information on tumor perfusion and tumor blood vessel functionality. ${ }^{13-16}$ Clinically relevant diagnostic modalities routinely used for anatomical, functional, and molecular angiogenesis imaging include contrastenhanced (Power Doppler) ultrasound, magnetic resonance imaging, and X-ray computed tomography (CT). Because of its high spatial resolution, reproducibility, user-friendliness, and suitability for high-throughput analyses, CT has become a routinely used method for noninvasively visualizing and quantifying the morphology and the functionality of tumor blood vessels. ${ }^{17,18}$ Preclinically, blood vessels are visualized and quantified mainly by use of contrast-enhanced volumetric CT or micro-CT $(\mu \mathrm{CT})$ approaches. ${ }^{16,19-23}$ In this regard, several studies using dynamic contrast-enhanced $\mu \mathrm{CT}$ imaging for the in vivo quantification of tumor angiogenesis and antiangiogenic therapy effects have been published recently, with assessment of vascular parameters such as tumor perfusion, tumor blood flow, vascular permeability, or relative blood volume (rBV). ${ }^{24-26}$ Importantly, however, there has been a lack of systematic study comparing the accuracy, the congruence, and the correlation of functional (steady-state) $\mu \mathrm{CT}$ imaging to IHC for determining $\mathrm{rBV}$ in tumors. In addition, in no previous studies has the anatomical information that can now be obtained at extremely high resolution by ex vivo $\mu \mathrm{CT}$ been used to quantitatively analyze the three-dimensional micromorphology of tumor blood vessels and to correlate it with the degree of tumor aggressiveness and angiogenesis.

Here, using four tumor models with different vascular characteristics and a combination of in vivo and ex vivo $\mu \mathrm{CT}$, we show that functional $\mu \mathrm{CT}$ imaging allows highly accurate quantification of the $\mathrm{rBV}$ in tumors, with values correlating very well with those obtained using IHC, and that anatomical imaging allows ultra-high-resolution structural analyses of the three-dimensional micromorphology of the tumor vasculature (Figure 1). Anatomical $\mu \mathrm{CT}$ can depict vessel diameters, vessel distribution, and vessel branching at sizes as small as $3.4 \mu \mathrm{m}$; thus, the visualization and quantification of such microvascular characteristics can be used to discriminate between tumors with low versus high degrees of aggressiveness and angiogenesis.

\section{Materials and Methods}

\section{Tumor Models}

Four different mouse xenograft models were used, based on A431 epidermoid carcinoma, Calu-6 anaplastic lung carcinoma, MLS ovarian carcinoma, and A549 non-small cell lung carcinoma human cell lines. All cell lines were obtained from the American Type Culture Collection (Manassas, VA);

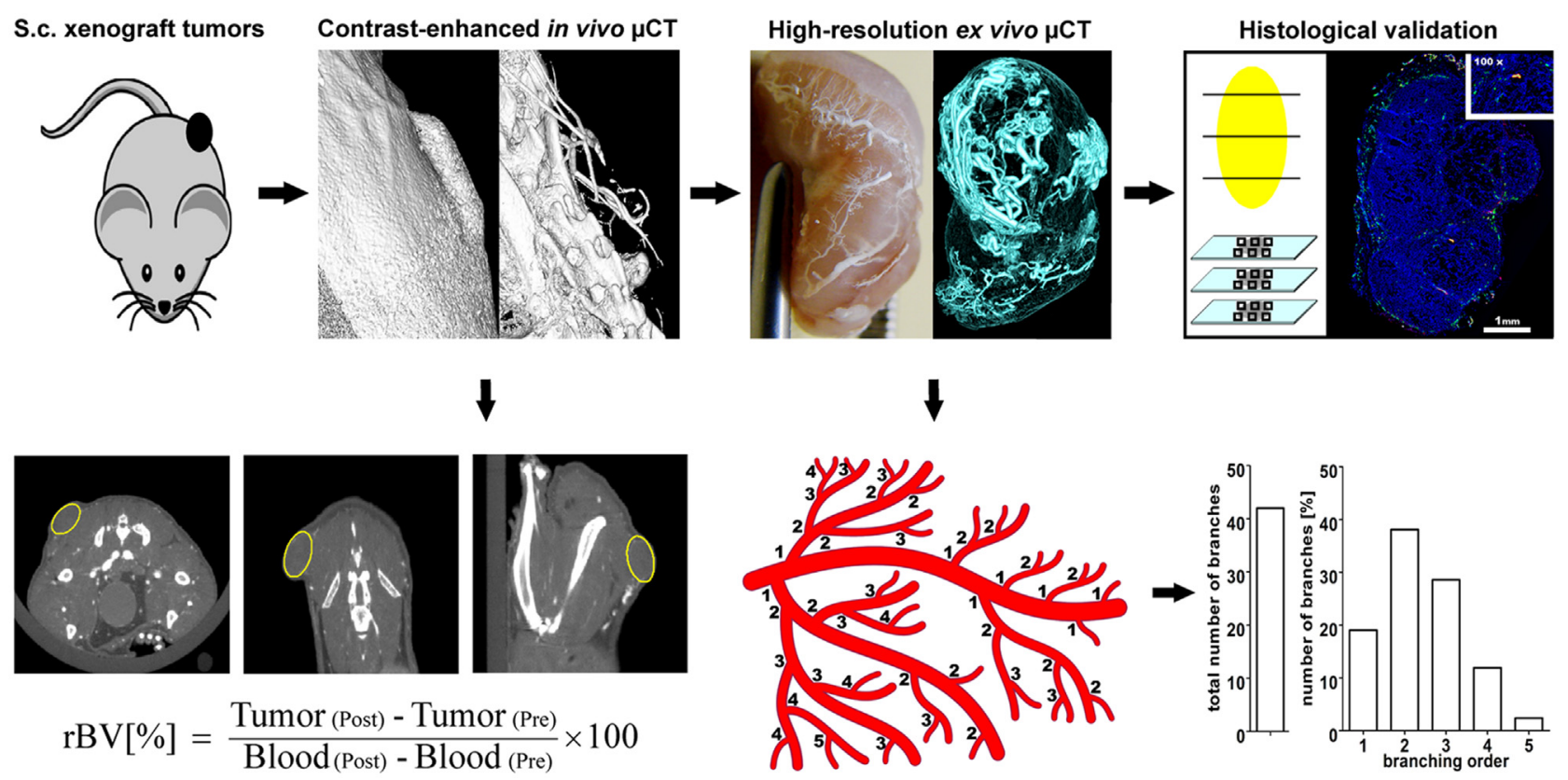

Figure 1 Study design. Four tumor models (A431, Calu-6, MLS, and A549) with differing degrees of angiogenesis were used to demonstrate the potential of combining in vivo and ex vivo $\mu \mathrm{CT}$ for highly accurate functional and ultra-high-resolution anatomical imaging of tumor angiogenesis. Using contrast-enhanced in vivo $\mu \mathrm{CT}$, the rBV in tumors approximately $6 \times 6 \mathrm{~mm}$ in size was determined noninvasively. The rBV values were then compared with those obtained with IHC by quantifying the area fraction of $\mathrm{CD} 31^{+}$blood vessels on three different sections for each tumor; both congruence and correlation were highly significant. After ex vivo imaging with Microfil perfusion, the three-dimensional micromorphology of tumor blood vessels was visualized at a resolution of approximately $3.4 \mu \mathrm{m}$; this allowed a highly detailed and quantitative analysis of the anatomical properties of the vascular network in tumors, as well as correlation of vessel size, vessel distribution, and vessel branching with the degree of angiogenesis by quantifying the amount of $\alpha \mathrm{SMA}^{+}$(mature) blood vessels using IHC. 
cells were resuscitated before use in in vivo experiments and were regularly tested for mycoplasma infection using Hoechst 33258 staining (1:400; Sigma-Aldrich, Steinheim, Germany) and fluorescence microscopy. Tumors were induced by inoculating $4 \times 10^{6}$ A431 cells, $2 \times 10^{6}$ Calu- 6 cells (in Matrigel; BD Biosciences, Heidelberg, Germany; San Jose, CA), $2.5 \times 10^{6}$ MLS cells or $3 \times 10^{6}$ A549 cells subcutaneously into the right flank of 8-week-old female CD1 nude mice (Charles River Laboratories International, Wilmington, MA) ( $n=5$ mice per model). Tumor growth was monitored by means of caliper measurements throughout the experimental period; tumor volumes were calculated as $(l \times$ $\left.w^{2}\right) / 2$, where $l$ is the largest diameter (length) and $w$ is the smallest diameter (width). Experiments were performed when tumors reached a volume of approximately $100 \mathrm{~mm}^{3}$. Animals were inhalation-anesthetized with $1.5 \%$ isofluorane in oxygen-enriched air with a face mask during all experimental procedures. All animal experiments were approved by the local and institutional ethics committees.

\section{In Vitro VEGF Production}

To determine VEGF production by the A431, Calu-6, MLS, and A549 human carcinoma cells, a human VEGF enzymelinked immunosorbent assay kit (ELISA; RayBiotech, Norcross, GA) was used. To this end, $2 \times 10^{5}$ cells were seeded in Petri dishes; 3 days later, when cells reached approximately $90 \%$ confluency, supernatants were harvested and transferred to an anti-human VEGF-coated microtiter plate, followed by application of horseradish peroxidaseconjugated streptavidin for 45 minutes and TMB One-Step substrate reagent (RayBiotech) for 30 minutes. Color intensity was determined at $450 \mathrm{~nm}$.

\section{In Vivo $\mu \mathrm{CT}$}

For in vivo $\mu \mathrm{CT}$, a dual-energy gantry-based flat-panel X-ray microtomography scanner was used (TomoScope 30s Duo; CT Imaging, Erlangen, Germany); this is a desktop cone-beam scanner with integrated radiation shielding, object diameter 40 $\mathrm{mm}$, two source-detector systems, and beam-hardening filter. Details of the scanning system and scanning protocol have been described previously. ${ }^{20}$ Tumor-bearing mice were scanned before and after intravenous administration of $100 \mu \mathrm{L}$ of an iodine-based blood-pool contrast agent (prepared inhouse) characterized by prolonged circulation kinetics and optimized for in vivo $\mu \mathrm{CT}$ imaging. ${ }^{27}$ The contrast agent was injected as a bolus into the lateral tail vein. Each in vivo $\mu \mathrm{CT}$ scan resulted in 2880 projections over a 6-minute time frame.

A Feldkamp-type reconstruction algorithm (CT Imaging), including ring artifact correction, was performed, with a voxel size of $35 \mu \mathrm{m}^{3}(35 \times 35 \times 35 \mu \mathrm{m})$. The reconstructed data sets were analyzed using Imalytics preclinical software version 1.2.1.4 (Philips Research, Aachen, Germany), which allows interactive segmentation and visualization of large data sets, including the adjustment of volumes of interest in any slicing orientation. After an elliptical volume of interest was defined, the $\mathrm{rBV}$ was determined based on the mean radiodensity, in Hounsfield units, of the segmented tumor and of a large reference blood vessel (the abdominal part of the aorta) before $(0 \% \mathrm{rBV})$ and after $(100 \% \mathrm{rBV})$ injection of the blood-pool contrast agent (Figure 1). The three-dimensional morphology of tumor blood vessels was visualized using MeVisLab software version 2.3.1 (MeVis Medical Solutions, Bremen, Germany).

\section{Ex Vivo $\mu \mathrm{CT}$}

After in vivo $\mu \mathrm{CT}$ imaging, tumor-bearing mice were intracardially perfused with Microfil injection compounds MV-112 and MV-Diluent (Flow Tech, Carver, MA), a leadcontaining silicone rubber $\mathrm{CT}$ contrast agent that polymerizes within the vascular compartment. ${ }^{28}$ Before Microfil perfusion, mice were perfused with $20 \mathrm{~mL}$ of PBS for complete blood removal and $20 \mathrm{~mL}$ of $4 \%$ paraformaldehyde for vessel fixation. Perfusion was performed by direct infusion into the left ventricle (after incising of the inferior vena cava) at physiological pressures, using a perfusion pump. After Microfil perfusion, tumors were excised and formalin-fixed.

The tumors were scanned using a non-gantry-based SkyScan $1172 \mu \mathrm{CT}$ system (SkyScan, Kontich, Belgium). Tumors were positioned on a computer-controlled rotation platform and were scanned 180 degrees around the vertical axis, in rotation steps of 0.3 degrees at $60 \mathrm{kV}$. Acquisition times ranged from 2 to 4 hours. The generated isotropic pixel sizes ranged from 3.4 to $5.3 \mu \mathrm{m}$. After three-dimensional volume rendering of reconstructed high-resolution $\mu \mathrm{CT}$ data sets, blood vessels were visualized using Imalytics preclinical software and vessel size as well as vessel distribution were analyzed in 10 randomly chosen fields of view (FOV $=$ $500 \times 350 \mu \mathrm{m})$ in the periphery versus the core of A431, Calu-6, MLS, and A549 xenografts. For each blood vessel, the diameter was determined in one of three possible planes (transverse, sagittal, or coronal) in which the cross-sectional area was relatively round. Values were compared with those obtained using IHC.

After threshold-based blood vessel segmentation, branching points and the three-dimensional micromorphology of tumor blood vessels were systematically analyzed using high-resolution ex vivo $\mu \mathrm{CT}$ and three-dimensional volume rendering. Similar thresholds were used for all tumors. To this end, all branching points within five representative vessels per FOV were manually counted, and the number of blood vessel branches per primary blood vessel was quantified (Figure 1).

\section{Immunohistochemistry}

Paraffin-embedded tumor sections were stained using CD31 (Dianova, Hamburg, Germany) and $\alpha$-SMA-biotin (PROGEN Biotechnik, Heidelberg, Germany) antibodies. Secondary antibodies were obtained from Dianova. Nuclei were 
counterstained using Hoechst nuclear dye, and sections were mounted using Mowiol polyvinyl alcohol medium. Fluorescence microscopy was performed using an Axio Imager M2 light microscope with an AxioCam MRm revision 3 high-resolution camera (both from Carl Zeiss Microimaging, Göttingen, Germany). For quantifying rBV, six images (three from the periphery and three from the core) were investigated $(\mathrm{FOV}=500 \times 350 \mu \mathrm{m})$, from three representative sections per tumor and five tumors per model. Both nonfilled $\mathrm{CD} 31^{+}$area fractions and semiautomatically filled $\mathrm{CD} 31^{+}$vascular structures were determined using a custom macro implemented for open-access image analysis software (ImageJ version 1.43u; NIH, Bethesda, MD) (Supplemental Figure S1). This approach, which has been described previously, ${ }^{29}$ is based on the detection and filling of $\mathrm{CD} 31^{+}$vascular structures. Vessel size was analyzed by determining diameters in 10 representative vessels in the tumor core versus periphery. To standardize the diameter quantifications in two-dimensional IHC slices, the shorter of two orthogonally arranged possible diameters was chosen. Images were analyzed and quantified using ImageJ software.

\section{Statistical Analysis}

Statistical and correlation analyses were performed using GraphPad Prism software version 5.0 (GraphPad Software, San Diego, CA). Two-tailed $t$-test and Fisher's $z$-test were used to assess statistical significance. $P<0.05$ was considered to represent statistical significance. Data are expressed as means $\pm \mathrm{SD}$.

\section{Results}

Biological and Immunohistochemical Characterization of the Tumor Models

Anatomical and functional CT imaging of tumor angiogenesis was performed in four mouse xenograft models with significantly different in vivo growth characteristics and vascularization: A431, Calu-6, MLS, and A549 tumors. Representative histopathological images for the four models are shown in Figure 2, A and B. The endothelial cell marker platelet endothelial cell adhesion molecule-1 (PECAM-1; alias CD31) provides information on blood vessel density and distribution, and the myofibroblast and pericyte marker $\alpha$-smooth muscle actin ( $\alpha$ SMA) provides information on blood vessel maturation. H\&E staining revealed that tumors did not contain large necrotic areas (such as could give rise to false-negative results on the rBV). Tumor growth, which generally correlates with tumor aggressiveness, was fastest for A431 tumors, followed by Calu- 6 and by MLS (Figure 2C). A549 tumors grew significantly more slowly than the other three xenograft models.

The total number of blood vessels per FOV was consistently highest in A431 tumors and lowest in A549 tumors (26 \pm 9 versus $9 \pm 2$ vessels per FOV, respectively) (Figure 2D), indicating that A431 tumors are not only more aggressive, but also more angiogenic than A549 tumors. In corroboration, the highest expression levels of VEGF were detected in A431 tumors, and the lowest levels in A549 (Supplemental Figure S2). Conversely, the overall degree of vessel maturation was highest in A549 and lowest in A431 tumors (73 \pm $11 \%$ versus $14 \pm 4 \% \propto \mathrm{SMA}^{+}$vessels, respectively) (Figure 2D). IHC analysis of the distribution of blood vessels within the A431, Calu-6, MLS, and A549 xenografts revealed that the tumors which intrinsically produce very high levels of VEGF (ie, A431 and MLS) were much more homogeneously vascularized than the tumors which express low levels of VEGF (ie, Calu-6 and A549), in which vessels were located mostly in the tumor periphery (Figure 2B).

\section{Anatomical and Functional in Vivo $\mu \mathrm{CT}$ Imaging of Tumor Angiogenesis}

Next, we visualized the three-dimensional micromorphology of tumor blood vessels in these four tumor models, and we quantified the $\mathrm{rBV}$ using in vivo dual-energy $\mu \mathrm{CT}$ and an iodine-containing contrast agent optimized for blood-pool imaging. ${ }^{15,20,30}$ Dual-source flat-panel in vivo $\mu \mathrm{CT}$ allowed noninvasive visualization of blood vessel branches up to the third order in MLS and A549 xenografts, whereas in the more aggressive and more angiogenic A431 and Calu-6 tumors only first-order blood vessel branches (in A431) or up to the second order (Calu-6) could be visualized (Figure 3, A-D). The resolution (ie, the smallest vessel size that could be noninvasively visualized) using this technique was approximately $40 \mu \mathrm{m}$.

To assess the accuracy of in vivo $\mu \mathrm{CT}$ imaging for quantitative angiogenesis characterization (relative to standard angiogenic profiling via IHC), noninvasively obtained rBV values were correlated with filled versus nonfilled area fractions of $\mathrm{CD} 31^{+}$vascular structures (Figure 4 and Supplemental Figure S1). To properly reflect differences in blood vessel distribution in the core versus the periphery of tumors, IHC images were obtained in an equal proportion: six images per section (three for the core and three for the periphery), three different sections per tumor, and five different tumors per model (Figure 1). rBV values obtained using in vivo $\mu \mathrm{CT}$ ranged from $2.6 \pm 0.5 \%$ (A549) to $6.0 \pm 1.5 \%$ (MLS); the intermediate values were $3.6 \pm 0.4 \%$ (Calu-6) and $4.7 \pm 0.9 \%$ (A431) (Figure 4A). IHC using filled CD31 ${ }^{+}$ vessel structures consistently rendered $\mathrm{rBV}$ values of $2.5 \pm$ $0.4 \%$ for A549, $3.2 \pm 0.5 \%$ for Calu- $6,4.1 \pm 1.2 \%$ for A 431 , and $5.0 \pm 1.4 \%$ for MLS (Figure 4A). A highly significant correlation between $\mathrm{rBV}$ values determined using in vivo $\mu \mathrm{CT}$ and IHC was observed, not only for all 20 tumors in total $(P<0.0001)$ (Figure 4B), but also for all four tumor models $(P=0.0239$ to 0.0019$)$ (Figure $4 \mathrm{C})$. These differences in functional blood vessels within the different tumor models are consistent with the in vitro VEGF levels (Supplemental Figure S2), indicating that contrast-enhanced in vivo $\mu \mathrm{CT}$ imaging not only can accurately determine small differences 

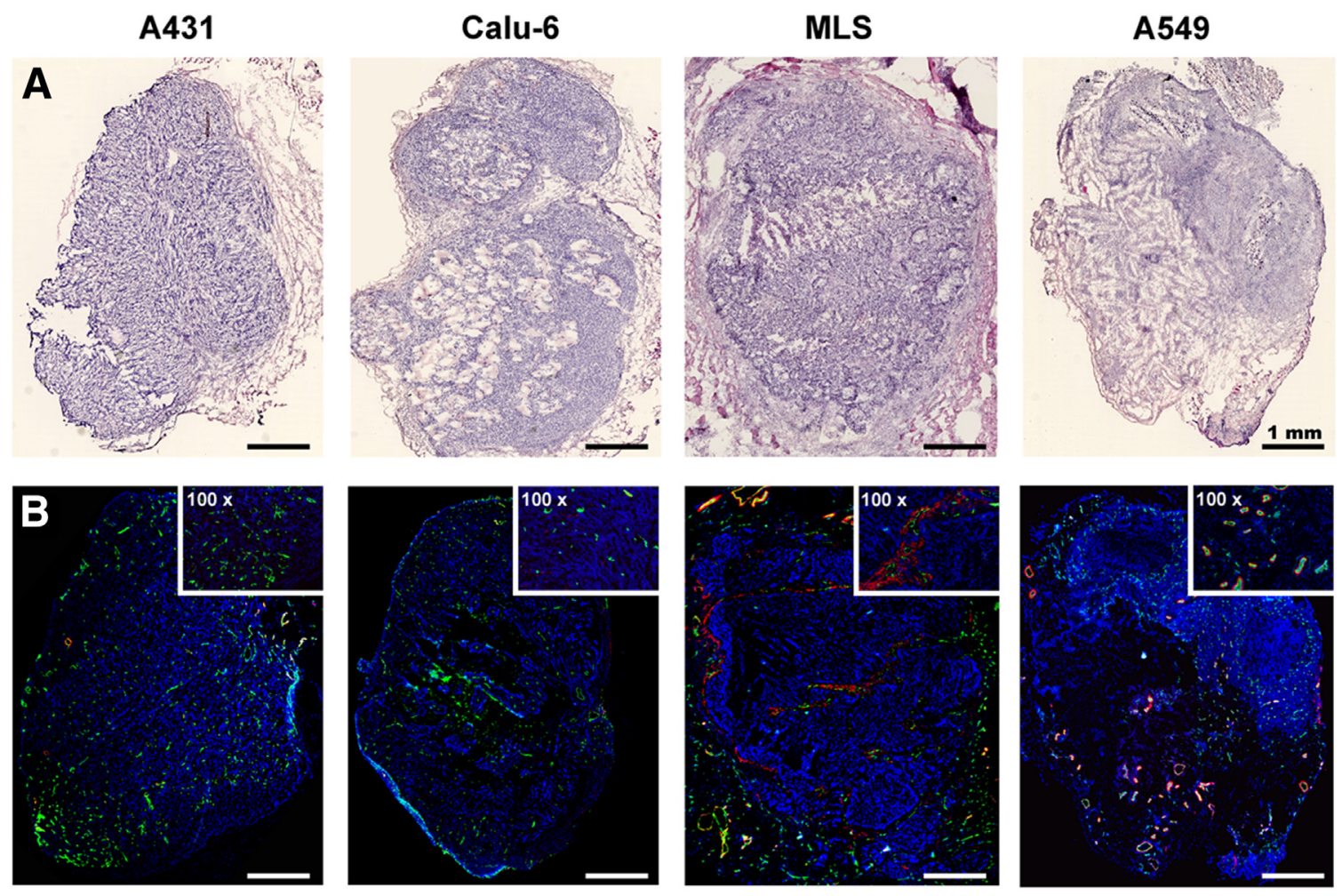

C
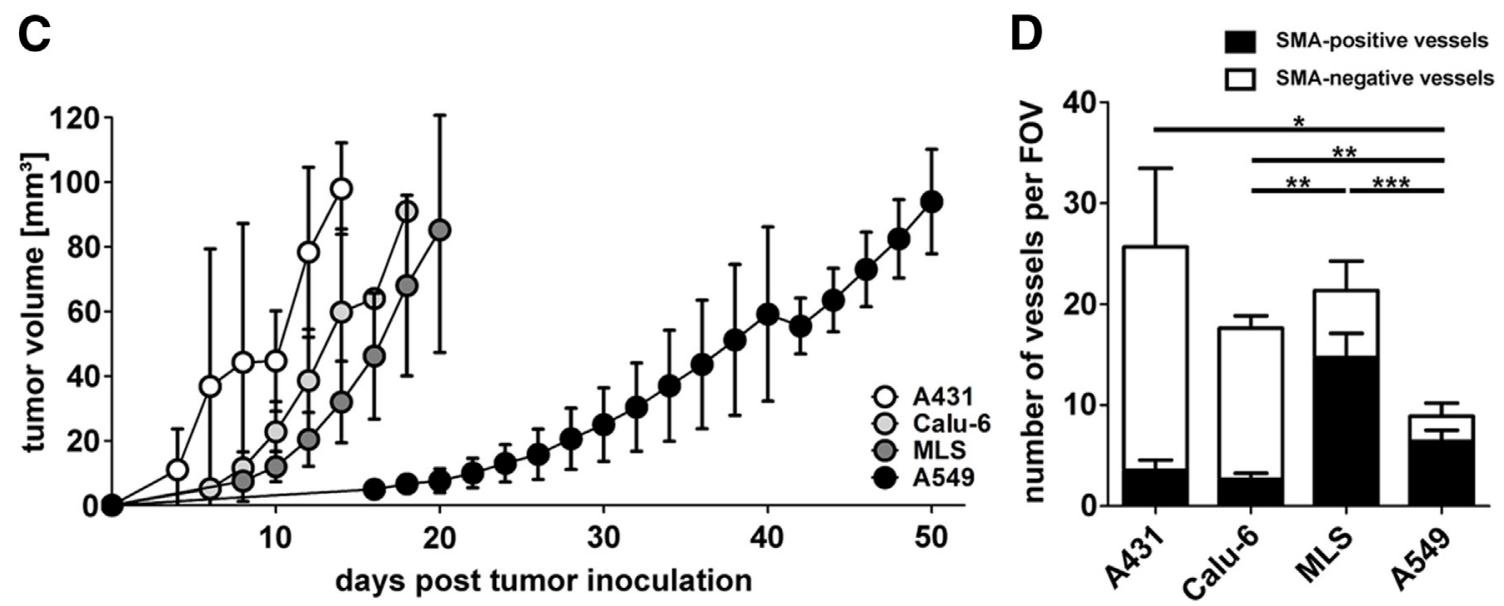

Figure 2 Characterization of tumor growth and angiogenesis. A and B: Representative histological and immunofluorescence images of A431, Calu-6, MLS, and A549 tumors with H\&E staining for histology (A) or with immunofluorescence staining (B) for CD31 (green) as a marker of blood vessels, for $\alpha$ SMA (red) as a marker of pericytes, and for Hoechst nuclear dye (blue). $\mathbf{C}$ and D: Differences in tumor growth (C) and blood vessel density and maturity (D) characterize the four models. Data are expressed as means \pm SD. $n=5$ tumors per model. ${ }^{*} P<0.05,{ }^{* *} P<0.01$, and ${ }^{* * *} P<0.001$, two-tailed $t$-test. Original magnification: $\times 100$ (B, insets).

in tumor vascularization, but also can quantitatively characterize the angiogenic activity of tumors with different growth characteristics. Finally, not filling the lumen of CD31 ${ }^{+}$ vascular structures in formalin-fixed tumor sections resulted in a significant underestimation of the $\mathrm{rBV}$ in IHC, with no correlation between in vivo $\mu \mathrm{CT}$ and IHC (Figure 4, D and E).

\section{Anatomical ex Vivo $\mu \mathrm{CT}$ Imaging of Tumor Angiogenesis}

These results convincingly demonstrate that in vivo $\mu \mathrm{CT}$ allows a highly accurate noninvasive determination of the
$\mathrm{rBV}$ in tumors. However, the spatial resolution of gantrybased in vivo $\mu \mathrm{CT}$ scanners ends at approximately $35 \mu \mathrm{m}$ per voxel (depending on scan protocols, scan times, biologically relevant X-ray doses, and contrast agents). ${ }^{16}$ The vast majority of newly formed blood vessels in tumors are smaller than $35 \mu \mathrm{m}$, but we overcame this limitation by visualizing and quantitatively characterizing the microarchitecture of vascular structures in Microfil-perfused A431, Calu-6, MLS, and A549 tumors using ultrahigh-resolution ex vivo $\mu \mathrm{CT}$ tomography. Representative three-dimensional images of ex vivo imaged blood vessel networks, as well as two-dimensional $\mu \mathrm{CT}$ images of the 

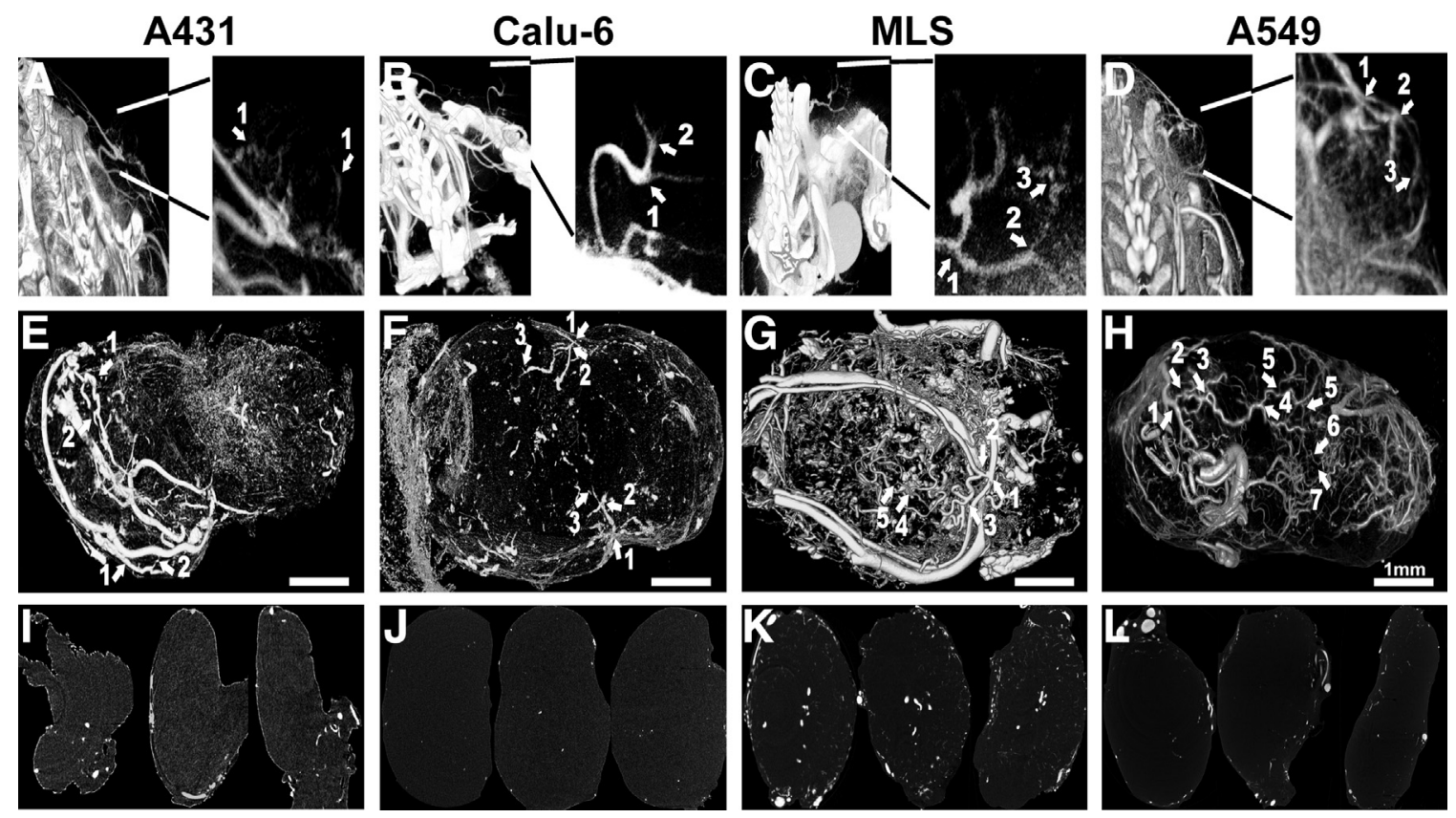

Figure 3 In vivo and ex vivo $\mu \mathrm{CT}$ imaging of tumor angiogenesis. A-D: Contrast-enhanced in vivo $\mu \mathrm{CT}$ was used for anatomical and functional visualization and quantitative characterization of tumor angiogenesis. Numbers represent the vessel branching order. In the xenograft models with large numbers of mature blood vessels (MLS and A549), blood vessel branches up to the third order could be visualized; in models characterized by more angiogenic and less mature blood vessels (A431 and Calu6), only first- and second-order blood vessel branches could be identified. The numbers within the images exemplify the rising order of vascular branches. E-H: After Microfil perfusion and vascular casting, ultra-high-resolution ex vivo $\mu \mathrm{CT}$ was performed with three-dimensional volume rendering, enabling the visualization and quantitative characterization of blood vessels with diameters as small as $3.4 \mu \mathrm{m}$, and blood vessel branches up to the seventh order. I-L: Two-dimensional cross-sectional images ( $x, y$, and $z$ planes) provide highly detailed information on blood vessel diameter, blood vessel density, and blood vessel distribution; tumors are the same as in panels $\mathbf{E}-\mathbf{H}$.

same tumors in transversal, sagittal, and coronal planes, are presented in Figure 3, E-L. Using this technique, blood vessels as small as $3.4 \mu \mathrm{m}$ could be visualized, and the three-dimensional vascular network in the xenograft models used could be visualized at very high resolution.

Microfil perfusion-based vascular casting combined with ex vivo $\mu \mathrm{CT}$ imaging allowed highly detailed threedimensional visualization of blood vessel distribution within the core and the periphery of tumors, as well as systematic and quantitative analysis of angiogenesis-related vascular parameters, such as vessel size, vessel distribution, and vessel branching (Figures 5 and 6). A technique assessing all of these parameters within intact tumors has not yet been described, but a quantitative characterization of the three-dimensional micromorphology of tumor blood vessels, especially with regard to their branching characteristics, would hold great potential for better understanding tumor angiogenesis and monitoring the efficacy of antiangiogenic therapies. In an empirical approach, we systematically investigated vessel size, vessel distribution, and vessel branching using Microfil perfusion-based vascular casting, high-resolution ex vivo $\mu \mathrm{CT}$, and volume-rendering techniques for quantitative characterization of tumor angiogenesis.

Consistent with their increased growth rate and higher degree of angiogenesis (Figures 2 and 3), a clear tendency toward reduced vessel size and reduced vessel branching was observed for A431 xenografts, relative to A549. Mean vessel size for A431 was $11 \pm 3 \mu \mathrm{m}$ (core) and $23 \pm 6 \mu \mathrm{m}$ (periphery), compared with $15 \pm 4 \mu \mathrm{m}$ and $35 \pm 11 \mu \mathrm{m}$, respectively, for A549. These values matched very well those obtained using IHC: $8 \pm 3 \mu \mathrm{m}$ (core) and $20 \pm 6 \mu \mathrm{m}$ (periphery) for $\mathrm{A} 431$, compared with $13 \pm 4 \mu \mathrm{m}$ and $32 \pm 13$ $\mu \mathrm{m}$, respectively, for A549 (Figure 5, A and B). Consistently, comparing vessel diameters obtained by ex vivo $\mu \mathrm{CT}$ and IHC confirmed a highly significant correlation between the two modalities $(P<0.0001)$ (Figure $5 \mathrm{C})$, demonstrating that ex vivo $\mu \mathrm{CT}$ is highly suitable for quantitative and ultrahigh-resolution anatomical analyses of tumor angiogenesis. Findings from qualitative and quantitative comparisons of blood vessels between exactly corresponding ex vivo $\mu \mathrm{CT}$ and IHC data sets of moderately vascularized A549 tumors and highly vascularized MLS tumors confirmed these findings (Figure 6). In addition, the percentages of blood vessels within a particular size range were analyzed using ex vivo $\mu \mathrm{CT}$; in the more aggressively growing and more angiogenic tumors (A431 and Calu-6), vessels were much smaller and much more homogeneous in size than in the less aggressively growing and less angiogenic tumors (MLS and A549) (Figure 5D).

Consistently, upon systematically analysis and quantification of the three-dimensional micromorphology of tumor blood vessels, more aggressive and more angiogenic tumors were found to have not only a much lower overall number of blood vessel branches, but also much lower orders of blood vessel branching (Figure 5, E and F). Quantification of the total number of branches per primary blood vessel in 

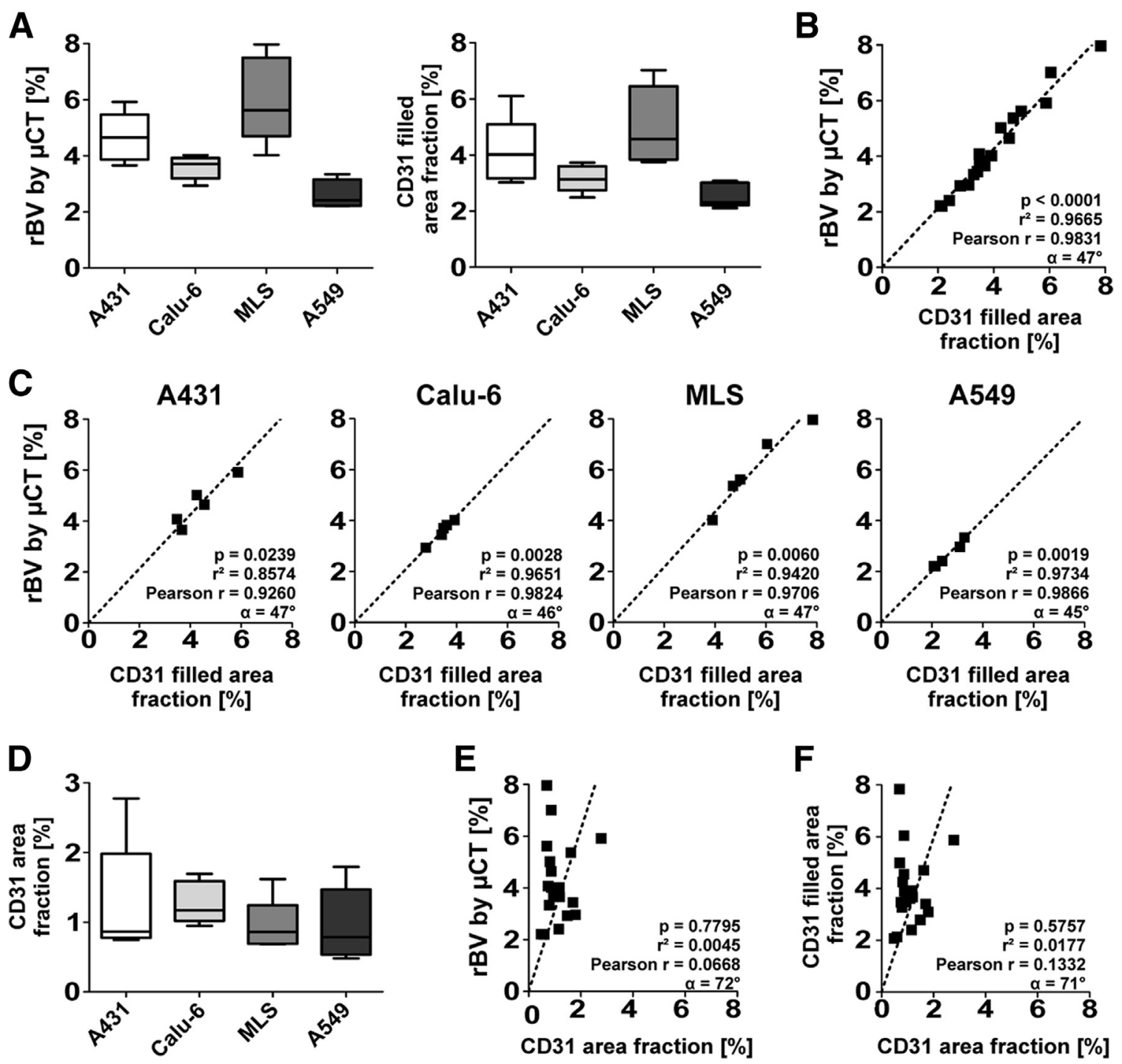

$\mathbf{F}$

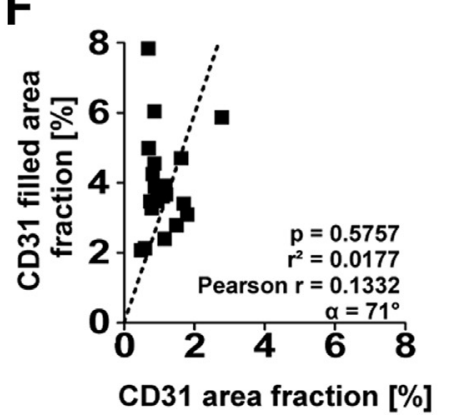

Figure 4 Congruence and correlation of functional in vivo $\mu \mathrm{CT}$ with IHC. A: Comparison of the model-dependent tumor rBV values determined using in vivo $\mu C T$ and IHC. To properly assess the rBV in IHC, the vessel lumen was semiautomatically filled and quantified in six different FOVs (three for the core and three for the periphery) in three representative sections from each of five tumors per tumor model. B: Correlation of the rBV values obtained on in vivo $\mu \mathrm{CT}$ and IHC after semiautomatic vessel filling. For the 20 tumors, the correlation was highly significant $(P<0.0001)$, and the linear regression $(\alpha=47$ degrees) indicates a highly accurate and congruent determination of the rBV by in vivo $\mu$ CT versus IHC. C: Correlation of the rBV values determined used in vivo $\mu$ CT versus IHC was significant for all four tumor models $(P<0.05)$. D: Quantification of nonfilled $\mathrm{CD} 31^{+}$area fractions in IHC, exemplifying that, compared with filled vessel structures and to in vivo $\mu \mathrm{CT}$, no significant differences were observed between the four tumor models used and that absolute rBV values are strongly underestimated. $\mathbf{E}$ and $\mathbf{F}$ : Correlation of nonfilled $\mathrm{CD}_{1} 1^{+}$area fractions in IHC with rBV values determined using in vivo $\mu \mathrm{CT}(\mathrm{E})$ and with semiautomatically filled CD31 area fractions (F), exemplifying poor congruence and correlation. Data are expressed as box plots \pm SD (A, C, and D).

A431, Calu-6, MLS, and A549 xenografts yielded values of 11 $\pm 5,19 \pm 6,52 \pm 37$, and $130 \pm 67$, respectively (Figure 5E). Analogously, analysis of the percentages of blood vessel branches of the first to the seventh order (Figure 1) revealed that, in the more rapidly growing and more angiogenic A431 and Calu- 6 tumors, the majority of vessels branched only once or twice, whereas in the more slowly growing and mature MLS and A549 tumors (Figure 2), a significant number of vessels branched four to seven times (Figure 5F). These findings exemplify the potential of using ultra-high-resolution ex vivo $\mu \mathrm{CT}$ for detailed visualization and quantitative characterization of the three-dimensional micromorphology of tumor blood vessels and for differentiating tumors with high versus low degrees of angiogenesis (Figure 3, E-H, and Supplemental Videos S1, S2, S3, and S4).

\section{Discussion}

Our aim was to systematically demonstrate that contrastenhanced in vivo $\mu \mathrm{CT}$ can be used to accurately quantify $\mathrm{rBV}$ in tumors, and to exemplify that ex vivo $\mu \mathrm{CT}$ allows quantitative and ultra-high-resolution analyses on the three-dimensional micromorphology and the angiogenic 

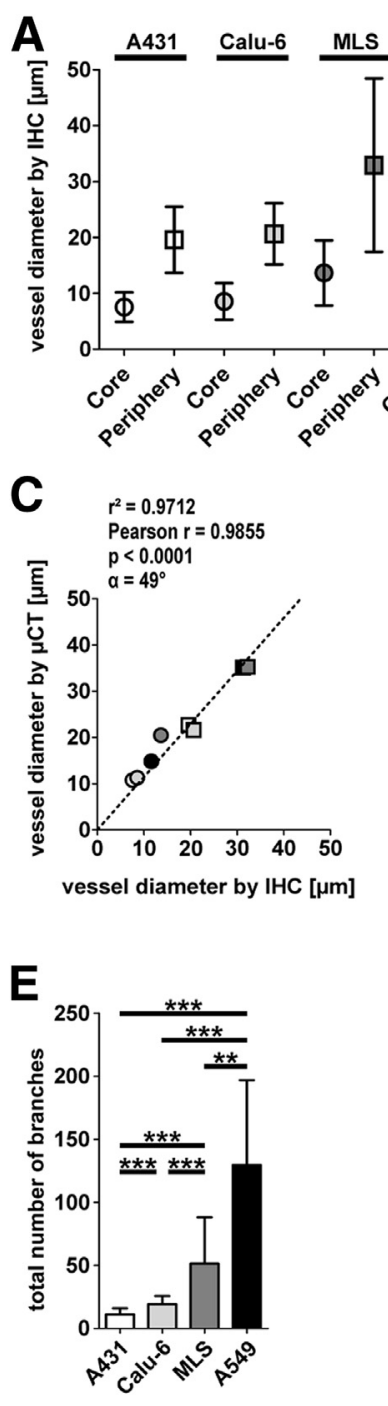
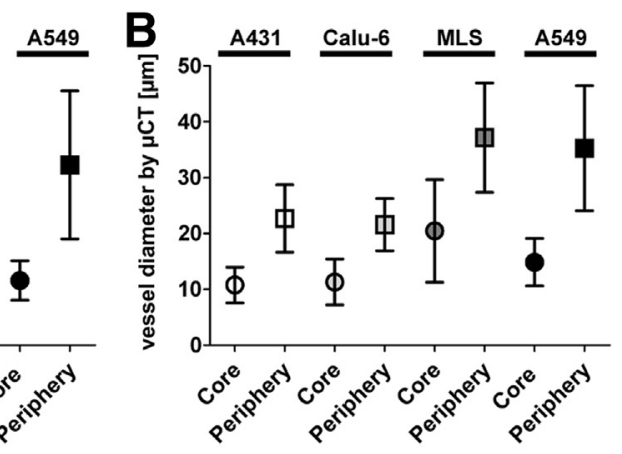

D 70

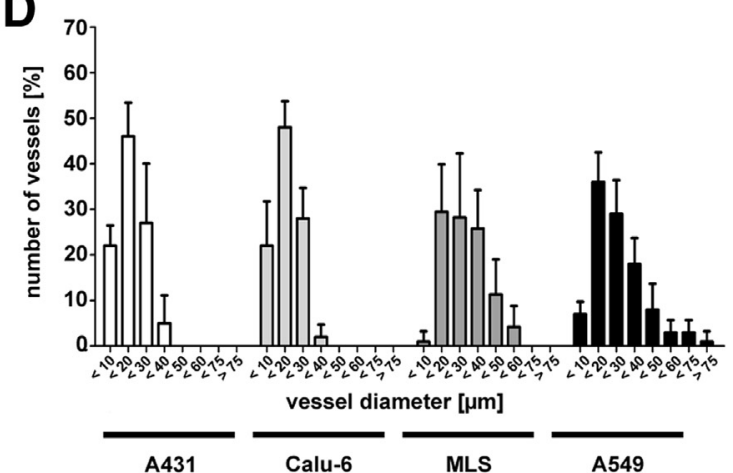

$\mathbf{F}$

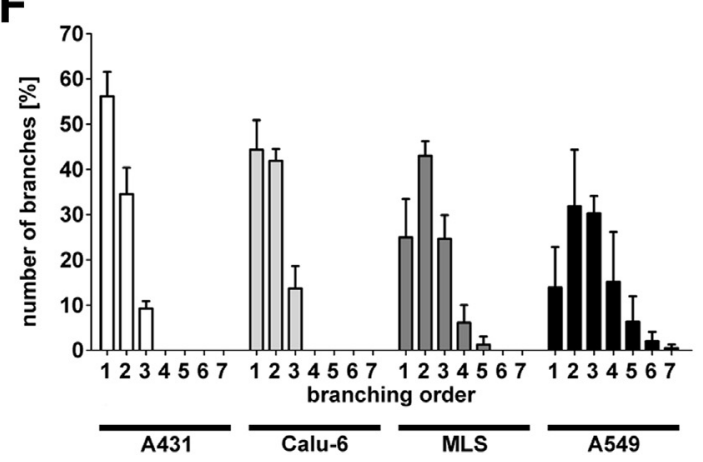

Figure 5 Quantitative ex vivo $\mu \mathrm{CT}$ imaging of tumor angiogenesis. A and B: Quantification of vessel diameters in the core and periphery of the four tumor models using IHC (A) and ex vivo $\mu \mathrm{CT}$ (B), exemplifying that vessels in more aggressive tumors (A431 and Calu-6), which are characterized by a more angiogenic and less mature vasculature, are smaller than vessels in less angiogenic and more mature tumors (MLS and A549). C: Correlation of vessel diameters determined using ex vivo $\mu \mathrm{CT}$ and IHC. D: Quantification of blood vessel diameter distribution within a particular size range, confirming that vessels in more aggressive and more angiogenic tumors were smaller and more homogeneous in size than in less aggressive and less angiogenic tumors. $\mathbf{E}$ and $\mathbf{F}$ : Ex vivo $\mu \mathrm{CT}$ based quantification of the total number of blood vessel branches (E) and of the percentage of branches by increasing order (F), exemplifying that less aggressive and more mature tumors (MLS and A549) contained more branches and higherorder branches per vessel than less mature tumors (A431 and Calu-6). Data are expressed as means $\pm \mathrm{SD}(\mathbf{A}, \mathbf{B}$, and $\mathbf{D}-\mathbf{F}) .{ }^{*} P<0.01,{ }^{* *} p<<$ 0.001 . characteristics of tumor blood vessels. To this end, using four different tumor models, we systematically compared the functional and anatomical information obtained using in vivo and ex vivo $\mu \mathrm{CT}$ with that obtained from IHC. The four xenograft models differed significantly in aggressiveness and angiogenesis. Rapidly growing A431 tumors contain many small and mostly immature blood vessels, which are homogeneously distributed throughout the tumor. Calu- 6 tumors are characterized by an intermediate growth rate and by an intermediate number of blood vessels, which are small, mostly immature and relatively heterogeneously distributed throughout the tumor; MLS tumors are also characterized by an intermediate growth rate, but (because of an extensive stromal compartment) the majority of blood vessels appear to be mature, and are distributed homogeneously throughout the tumor. Slowly growing A549 tumors contain a relatively small number of blood vessels, the vast majority of which are large and mature, and are distributed relatively heterogeneously throughout the tumor (Figure 2). With these four models, we show that contrast-enhanced in vivo $\mu \mathrm{CT}$ is an excellent tool for noninvasively visualizing and quantifying the rBV in tumors. Together with the highly detailed anatomical information that can be obtained about the three-dimensional microvascular network within tumors using in vivo and ex vivo $\mu \mathrm{CT}$, these findings indicate that $\mu \mathrm{CT}$ is a highly useful and highly versatile tool for characterizing tumor angiogenesis.

Our present findings extend previous efforts on anatomical and functional $\mu \mathrm{CT}$ imaging of tumor angiogenesis in several ways. First, in terms of functional in vivo $\mu \mathrm{CT}$, in no previous studies have $\mathrm{rBV}$ values obtained noninvasively using steady-state $\mu \mathrm{CT}$ been quantitatively and systematically compared with those obtained using IHC in multiple xenograft models differing in their vascular characteristics. Several previous analyses have shown a correlation between the rBV determined using dynamic $(\mu) \mathrm{CT}$ or steady-state magnetic resonance imaging and the microvessel density determined using IHC, both in animal models and in patients, ${ }^{24-26,31-37}$ but the present study is the first to systematically demonstrate a highly significant congruence 

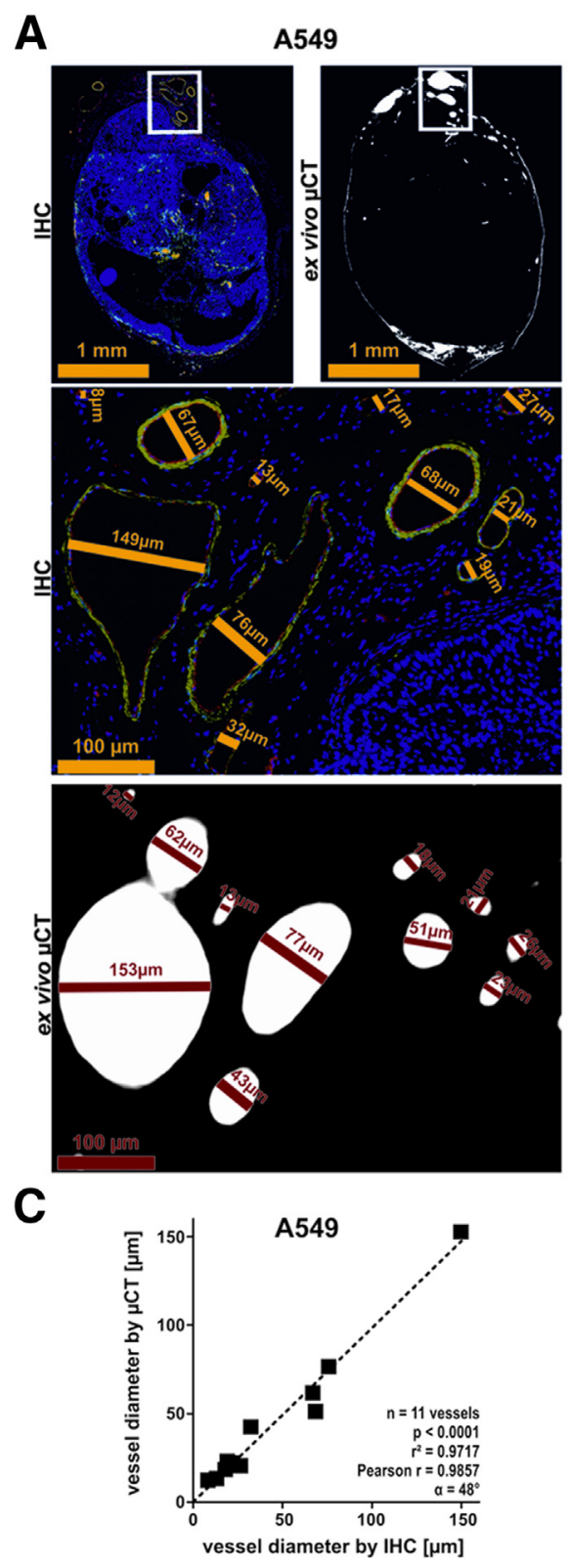

B

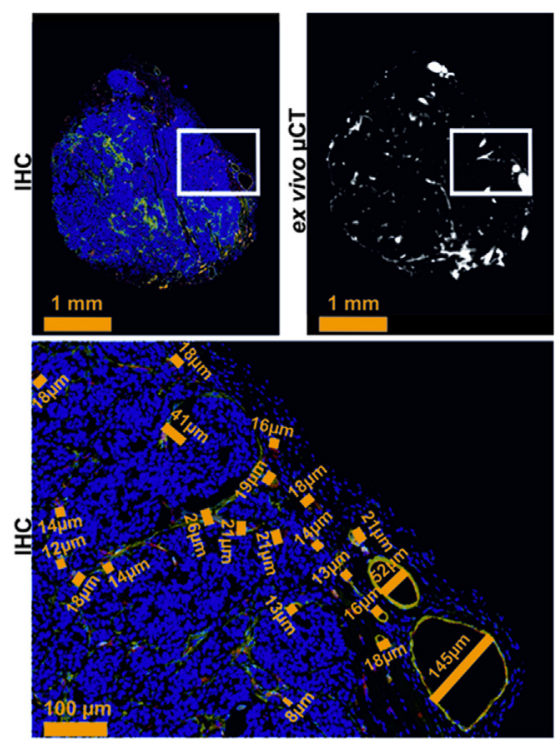

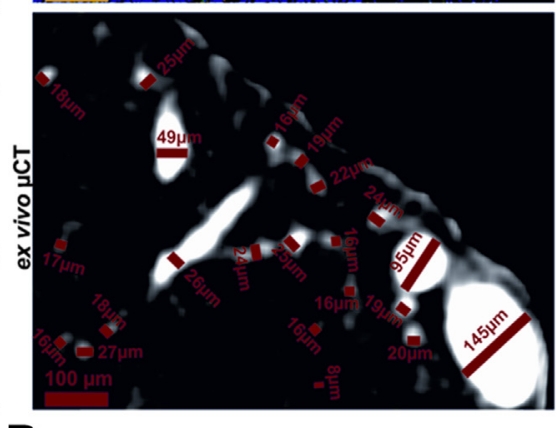

D

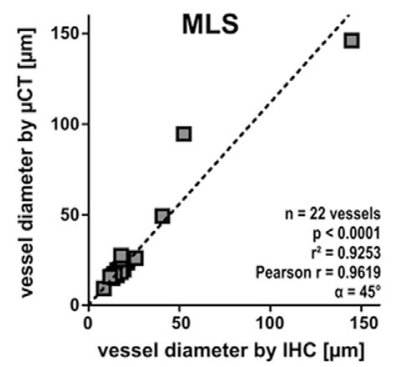

Figure 6 Qualitative and quantitative comparison of tumor blood vessels in corresponding ex vivo $\mu \mathrm{CT}$ and IHC data sets. A and B: Immunofluorescence images and corresponding two-dimensional crosssectional images from high-resolution ex vivo $\mu \mathrm{CT}$ imaging after Microfil perfusion and vascular casting of moderately vascularized A549 (A) and highly vascularized MLS (B) tumors. Boxed regions in the whole-tumor images (top row) are shown at higher magnification in the middle and bottom rows. Immunohistochemical staining was performed using antibodies against CD31 (blood vessels; red), aSMA (pericytes; green), and Hoechst nuclear dye (blue). C and $\mathbf{D}$ : Correlation of vessel diameters determined using corresponding IHC and ex vivo $\mu \mathrm{CT}$ data sets, exemplifying a highly significant correlation $(P<$ $0.0001)$ and linear regression $\alpha$ values close to 45 degrees for both A549 (C) and MLS (D) tumors. between $\mathrm{rBV}$ values determined using three-dimensional steady-state $\mu \mathrm{CT}$ and two-dimensional IHC (Figure 4). Second, in terms of anatomical $\mu \mathrm{CT}$ imaging, we for the first time provide ex vivo images of the vascular network in subcutaneous tumor xenografts at a resolution of less than 4 $\mu \mathrm{m}$. These images exemplify the huge potential of vascular casting and ultra-high-resolution ex vivo $\mu \mathrm{CT}$ for quantitatively analyzing the three-dimensional micromorphology of tumor blood vessels (Figure 3). Third, we demonstrate for the first time that blood vessel diameters, blood vessel distribution, and blood vessel branching in tumors can be accurately and quantitatively analyzed using ex vivo $\mu \mathrm{CT}$, with two-dimensional imaging information on vessel size and vessel distribution corresponding very well with that observed in IHC and with three-dimensional information on blood vessel branching corresponding very well with the degree of aggressiveness and angiogenesis of the different tumor models used (Figure 5). Importantly in this regard, the application of architectural and functional vessel imaging to identify, monitor, and predict outcomes for cancer patients who respond to antiangiogenic therapies has been described. ${ }^{38,39}$ In the present study, we could not perform validation of the high-resolution $\mu \mathrm{CT}$-based branching analyses using the acquired IHC data set, because of the two-dimensionality of conventional histological procedures. This limitation could be overcome by using advanced multiphoton or super-resolution microscopy techniques, which we plan to address in future studies.

Compared with other clinically relevant imaging modalities that can be used for visualizing and analyzing tumor angiogenesis, such as ultrasound and magnetic resonance imaging, ${ }^{13,40-42}$ combining in vivo and ex vivo $\mu \mathrm{CT}$ 
imaging has both advantages and disadvantages. The advantages include observer-independence, user-friendliness, the quantitative nature for both functional and micromorphological tumor blood vessel characterization, low costs, and high resolution, as well as suitability for high-throughput analyses. The disadvantages include X-ray exposure, the need for contrast agents, and the relatively low contrast-agent sensitivity of CT. ${ }^{13,16-19}$ In particular, in longitudinal studies assessing effects of antiangiogenic and/or antitumorigenic therapy, appropriate X-ray doses that do not affect tumor biology and tumor development should be taken into account, along with the individual accumulated load for iodinebased contrast agents.

Overall, when balancing these various pros and cons, and taking into account the findings reported here, it can be concluded that $\mu \mathrm{CT}$ is a simple, rational, reliable, and broadly applicable tool for visualizing and quantifying tumor angiogenesis, in particular in small-animal models such as mouse or rat. This is not only because $\mu \mathrm{CT}$ provides rapid and highly detailed anatomical information on the size and shape of tumors, but also because it allows highthroughput, highly accurate, and highly quantitative functional analyses on the $\mathrm{rBV}$ in tumors. In addition, with respect to pathophysiological conditions of circulation in tumors, $\mu \mathrm{CT}$ can be used to visualize and quantitatively characterize the three-dimensional micromorphology of tumor blood vessels, thereby differentiating between tumors with high versus low degrees of aggressiveness and angiogenesis. Consequently, the combination of anatomical and functional $\mu \mathrm{CT}$ seems to hold significant potential for facilitating translational anticancer research.

\section{Acknowledgments}

MeVis Medical Solutions AG (Bremen, Germany) and Philips Research (Aachen, Germany) are acknowledged for software support.

\section{Supplemental Data}

Supplemental material for this article can be found at http://dx.doi.org/10.1016/j.ajpath.2013.10.014.

\section{References}

1. Folkman J: Angiogenesis in cancer, vascular, rheumatoid and other disease. Nat Med 1995, 1:27-31

2. Carmeliet P, Jain RK: Angiogenesis in cancer and other diseases. Nature 2000, 407:249-257

3. Carmeliet P, Jain RK: Molecular mechanisms and clinical applications of angiogenesis. Nature 2011, 473:298-307

4. Folkman J: Tumor angiogenesis: therapeutic implications. N Engl J Med 1971, 285:1182-1186

5. Hanahan D, Weinberg RA: Hallmarks of cancer: the next generation Cell 2011, 144:646-674

6. Cao Y, Arbiser J, D'Amato RJ, D'Amore PA, Ingber DE, Kerbel R, Klagsbrun M, Lim S, Moses MA, Zetter B, Dvorak H, Langer R
Forty-year journey of angiogenesis translational research. Sci Transl Med 2011, 3:114rv3

7. Jain RK, Carmeliet P: SnapShot: Tumor angiogenesis. Cell 2012, 149: 1408-1408.e1

8. Weidner N, Semple JP, Welch WR, Folkman J: Tumor angiogenesis and metastasis-correlation in invasive breast carcinoma. N Engl J Med 1991, 324:1-8

9. Hlatky L, Hahnfeldt P, Folkman J: Clinical application of antiangiogenic therapy: microvessel density, what it does and doesn't tell us. J Natl Cancer Inst 2002, 94:883-893

10. Bourboulia D, Jensen-Taubman S, Rittler MR, Han HY, Chatterjee T, Wei B, Stetler-Stevenson WG: Endogenous angiogenesis inhibitor blocks tumor growth via direct and indirect effects on tumor microenvironment. Am J Pathol 2011, 179:2589-2600

11. Bzyl J, Palmowski M, Rix A, Arns S, Hyvelin JM, Pochon S, Ehling J, Schrading S, Kiessling F, Lederle W: The high angiogenic activity in very early breast cancer enables reliable imaging with VEGFR2targeted microbubbles (BR55). Eur Radiol 2013, 23:468-475

12. Zwick S, Strecker R, Kiselev V, Gall P, Huppert J, Palmowski M, Lederle W, Woenne EC, Hengerer A, Taupitz M, Semmler W, Kiessling F: Assessment of vascular remodeling under antiangiogenic therapy using DCE-MRI and vessel size imaging. J Magn Reson Imaging 2009, 29:1125-1133

13. McDonald DM, Choyke PL: Imaging of angiogenesis: from microscope to clinic. Nat Med 2003, 9:713-725

14. Vakoc BJ, Lanning RM, Tyrrell JA, Padera TP, Bartlett LA, Stylianopoulos T, Munn LL, Tearney GJ, Fukumura D, Jain RK, Bouma BE: Three-dimensional microscopy of the tumor microenvironment in vivo using optical frequency domain imaging. Nat Med 2009, 15:1219-1223

15. Jain RK, Munn LL, Fukumura D: Dissecting tumour pathophysiology using intravital microscopy. Nat Rev Cancer 2002, 2:266-276

16. Ehling J, Lammers T, Kiessling F: Non-invasive imaging for studying anti-angiogenic therapy effects. Thromb Haemost 2013, 109:375-390

17. Miller JC, Pien HH, Sahani D, Sorensen AG, Thrall JH: Imaging angiogenesis: applications and potential for drug development. J Natl Cancer Inst 2005, 97:172-187

18. Lee TY, Purdie TG, Stewart E: CT imaging of angiogenesis. Q J Nucl Med 2003, 47:171-187

19. Kiessling F, Greschus S, Lichy M, Bock M, Fink C, Vosseler S, Moll J, Mueller MM, Fusenig NE, Traupe H, Semmler W: Volumetric computed tomography (VCT): a new technology for noninvasive, high-resolution monitoring of tumor angiogenesis. Nat Med 2004, 10: $1133-1138$

20. Gremse F, Grouls C, Palmowski M, Lammers T, de Vries A, Grüll H, Das M, Mühlenbruch G, Akhtar S, Schober A, Kiessling F: Virtual elastic sphere processing enables reproducible quantification of vessel stenosis at CT and MR angiography. Radiology 2011, 260:709-717

21. Schambach SJ, Bag S, Steil V, Isaza C, Schilling L, Groden C, Brockmann MA: Ultrafast high-resolution in vivo volume-CTA of mice cerebral vessels. Stroke 2009, 40:1444-1450

22. Figueiredo G, Brockmann C, Boll H, Heilmann M, Schambach SJ, Fiebig T, Kramer M, Groden C, Brockmann MA: Comparison of digital subtraction angiography, micro-computed tomography angiography and magnetic resonance angiography in the assessment of the cerebrovascular system in live mice. Clin Neuroradiol 2012, 22:21-28

23. Sawall S, Kuntz J, Socher M, Knaup M, Hess A, Bartling S, Kachelrieß M: Imaging of cardiac perfusion of free-breathing small animals using dynamic phase-correlated micro-CT. Med Phys 2012, 39:7499-7506

24. Park CM, Goo JM, Lee HJ, Kim MA, Kim HC, Kim KG, Lee CH, Im JG: FN13762 murine breast cancer: region-by-region correlation of first-pass perfusion CT indexes with histologic vascular parameters. Radiology 2009, 251:721-730

25. Tai JH, Tessier J, Ryan AJ, Hoffman L, Chen X, Lee TY: Assessment of acute antivascular effects of vandetanib with high-resolution dynamic contrast-enhanced computed tomographic imaging in a human 
colon tumor xenograft model in the nude rat. Neoplasia 2010, 12: 697-707

26. Eisa F, Brauweiler R, Hupfer M, Nowak T, Lotz L, Hoffmann I, Wachter D, Dittrich R, Beckmann MW, Jost G, Pietsch H, Kalender WA: Dynamic contrast-enhanced micro-CT on mice with mammary carcinoma for the assessment of antiangiogenic therapy response. Eur Radiol 2012, 22:900-907

27. De Vries A, Custers E, Lub J, van den Bosch S, Nicolay K, Grüll H: Block-copolymer-stabilized iodinated emulsions for use as CT contrast agents. Biomaterials 2010, 31:6537-6544

28. Savai R, Langheinrich AC, Schermuly RT, Pullamsetti SS, Dumitrascu R, Traupe H, Rau WS, Seeger W, Grimminger F, Banat GA: Evaluation of angiogenesis using micro-computed tomography in a xenograft mouse model of lung cancer. Neoplasia 2009, 11: $46-56$

29. Tong RT, Boucher Y, Kozin SV, Winkler F, Hicklin DJ, Jain RK: Vascular normalization by vascular endothelial growth factor receptor 2 blockade induces a pressure gradient across the vasculature and improves drug penetration in tumors. Cancer Res 2004, 64:3731-3736

30. Kalender WA, Kyriakou Y: Flat-detector computed tomography (FDCT). Eur Radiol 2007, 17:2767-2779

31. d'Assignies G, Couvelard A, Bahrami S, Vullierme MP, Hammel P, Hentic O, Sauvanet A, Bedossa P, Ruszniewski P, Vilgrain V: Pancreatic endocrine tumors: tumor blood flow assessed with perfusion CT reflects angiogenesis and correlates with prognostic factors. Radiology 2009, 250:407-416

32. Persigehl T, Wall A, Kellert J, Ring J, Remmele S, Heindel W, Dahnke H, Bremer C: Tumor blood volume determination by using susceptibility-corrected DeltaR2* multiecho MR. Radiology 2010, 255:781-789

33. Remmele S, Ring J, Sénégas J, Heindel W, Mesters RM, Bremer C, Persigehl T: Concurrent MR blood volume and vessel size estimation in tumors by robust and simultaneous $\Delta \mathrm{R} 2$ and $\Delta \mathrm{R} 2 *$ quantification. Magn Reson Med 2011, 66:144-153
34. Ring J, Persigehl T, Remmele S, Heindel W, Dahnke H, Bremer C: Monitoring of bevacizumab-induced antiangiogenic treatment effects by "steady state" ultrasmall superparamagnetic iron oxide particles magnetic resonance imaging using robust multiecho $\Delta \mathrm{R} 2 *$ relaxometry. Invest Radiol 2011, 46:326-330

35. Ippolito D, Capraro C, Casiraghi A, Cestari C, Sironi S: Quantitative assessment of tumour associated neovascularisation in patients with liver cirrhosis and hepatocellular carcinoma: role of dynamic-CT perfusion imaging. Eur Radiol 2012, 22:803-811

36. Miles KA, Lee TY, Goh V, Klotz E, Cuenod C, Bisdas S, Groves AM, Hayball MP, Alonzi R, Brunner T; Experimental Cancer Medicine Centre Imaging Network Group: Current status and guidelines for the assessment of tumour vascular support with dynamic contrastenhanced computed tomography. Eur Radiol 2012, 22:1430-1441

37. Ren Y, Fleischmann D, Foygel K, Molvin L, Lutz AM, Koong AC, Jeffrey RB, Tian L, Willmann JK: Antiangiogenic and radiation therapy: early effects on in vivo computed tomography perfusion parameters in human colon cancer xenografts in mice. Invest Radiol 2012, 47:25-32

38. Emblem KE, Mouridsen K, Bjornerud A, Farrar CT, Jennings D, Borra RJ, Wen PY, Ivy P, Batchelor TT, Rosen BR, Jain RK, Sorensen AG: Vessel architectural imaging identifies cancer patient responders to anti-angiogenic therapy. Nat Med 2013, 19:1178-1183

39. Jiang T, Kambadakone A, Kulkarni NM, Zhu AX, Sahani DV: Monitoring response to antiangiogenic treatment and predicting outcomes in advanced hepatocellular carcinoma using image biomarkers, CT perfusion, tumor density, and tumor size (RECIST). Invest Radiol 2012, 47:11-17

40. Lindner JR: Microbubbles in medical imaging: current applications and future directions. Nat Rev Drug Discov 2004, 3:527-532

41. Barrett T, Brechbiel M, Bernardo M, Choyke PL: MRI of tumor angiogenesis. J Magn Reson Imaging 2007, 26:235-249

42. Weissleder R, Pittet MJ: Imaging in the era of molecular oncology. Nature 2008, 452:580-589 